\title{
Repercussões do tempo operatório em pulmões de ratos idosos ${ }^{1}$
}

\author{
Aldo da Cunha Medeiros ${ }^{2}$ \\ Keyla Borges Ferreira Rocha ${ }^{3}$ \\ Antônio Medeiros Dantas Filho ${ }^{4}$ \\ Tertuliano Aires Neto ${ }^{4}$ \\ Francisco Edilson Leite Pinto $\mathrm{Jr}^{4}$ \\ Bruno Cunha Medeiros ${ }^{5}$
}

\begin{abstract}
Medeiros AC, Rocha KBF, Dantas Filho AM, Aires Neto T, Pinto Jr FEL, Medeiros BC. Repercussões histopatológicas do tempo operatório em pulmões de ratos idosos. Acta Cir Bras [online] 2003 vol 18 suppl 1. Disponível em www.scielo.br/acb.
\end{abstract}

RESUMO: A duração das operações pode representar um fator importante para uma série de complicações pós-operatórias, especialmente para os indivíduos idosos. Objetivo: estudar a repercussão nos pulmões, de operações de diferentes tempos de duração. Métodos: Vinte ratos idosos (18 meses) e 20 jovens ( 3 meses) foram separados aleatoriamente em grupos A e B respectivamente. Os grupos foram divididos em A1, A2, A3, A4, B1, B2, B3 and B4, com cinco ratos cada. Os animais foram anestesiados com pentobarbital $(20 \mathrm{mg} / \mathrm{Kg})$ intraperitoneal. No subgrupo A1 e B1 foi feita operação com duração de 30 minutos, nos grupos A2 and B2 60 minutos, em A3 and B3 a operação foi feita em 120 minuto e os animais A4 e B4 (controle) não foram operados. O procedimento consistiu de laparotomia xifopubiana que foi aberta e fechada tantas vezes quanto necessário para atingir os tempos estipulados. Após o quinto dia pós-operatório os animais foram mortos com superdose de anestésico e biópsias de ambos os pulmões foram realizadas. Os achados histopatológicos foram transformados em escores. Resultados: os grupos de ratos jovens atingiram os escores: $\mathrm{A} 1=$ escore $6, \mathrm{~A} 2=11 ; \mathrm{A} 3=28 ; \mathrm{A} 4=5$. Os ratos idosos tiveram os escores: $\mathrm{B} 1=12$; $\mathrm{B} 2=34 ; \mathrm{B} 3=51$ e $\mathrm{B} 4=6$. A análise estatística revelou diferenças significantes entre os escores dos grupos A e B. Conclusões: $\mathrm{O}$ tempo prolongado nas operações realizadas em ratos idosos contribuiu para o aparecimento de alterações pulmonares de modo significante. Quanto maior o tempo operatório, mais intensas e mais freqüentes as complicações pulmonares.

DESCRITORES: Complicações pulmonares. Idoso. Tempo operatório. Ratos idosos.

\section{INTRODUÇÃO}

O envelhecimento afeta todos os órgãos, surgindo seqüelas funcionais variáveis que têm impacto negativo na resposta dos pacientes idosos ao estresse cirúrgico. Portanto, é obrigatório que sejam compreendidas e reconhecidas as alterações fisiológicas que acontecem nos vários órgãos com o envelhecimento ${ }^{1}$.

Em circunstâncias normais e sem estresse, as alterações fisiológicas que ocorrem no coração, nos pulmões, nos rins e no tracto gastrointestinal durante o envelhecimento, determinam deficiência insignificante

1. Trabalho realizado no Núcleo de Cirurgia Experimental-UFRN; Apoiado pelo CNPq.

2. Doutor em Cirurgia; Prof. Adjunto coordenador da Disciplina de Técnica Operatória- UFRN, Chefe do Núcleo de Cirurgia Experimental; Pesquisador nível 1 do CNPq

3. Prof. Mestre do Departamento de Patologia-UFRN

4. Prof. do Departamento de Cirurgia, aluno do Programa de Pós-graduação

5. Aluno do Programa de Iniciação Científica. 
de função. Entretanto, no estresse decorrente do ato operatório ou da doença aguda, a resposta do paciente idoso ao trauma diminui significativamente, em virtude da pequena reserva orgânica. Essa diminuição na reserva fisiológica, associada à idade, faz com que haja um maior risco de mortalidade, morbidez e complicações pós-operatórias ${ }^{2,3,4,5,6,7}$.

A duração das operações pode representar um fator a mais para uma série de complicações pós-operatórias tanto em indivíduos jovens quanto em idosos ${ }^{8}$. Está comprovado que cada hora adicional no tempo das operações representa o dobro da incidência de infecção pós-operatória, além de maior morbidez e mortalidade? $\mathrm{O}$ presente trabalho tem o objetivo de estudar as repercussões de operações de diferentes tempos de duração nos pulmões de ratos com 18 meses de idade, comparadas com animais jovens.

\section{MÉTODOS}

Foram utilizados 40 ratos Wistar sendo, 20 animais com 3 meses (grupo A) com peso $167 \pm 21 \mathrm{~g}$ e 20 outros com 18 meses de idade (grupo B), pesando $340 \pm 15 \mathrm{~g}$, separados aleatoriamente em 8 subgrupos de 5 animais cada. Foram anestesiados com pentobarbital sódico na dose de $20 \mathrm{mg} / \mathrm{Kg}$ por via intraperitoneal, depilação ampla da parede abdominal, antissepsia com PVPI 1\%, e laparotomia mediana xifopubiana. Nos subgrupos A1 e B-1 a duração da operação foi de 30 minutos; nos grupos A-2 e B-2 foi de 60 minutos e nos subgrupos A-3 e B-3 a operação se prolongou por 120 minutos. O subgrupos A-4 e B-4 (controle) não foram operados. $\mathrm{O}$ procedimento constou de abertura e fechamento da laparotomia tantas vezes quanto necessárias para completar os respectivos tempos operatórios, com sutura por planos, em que foi utilizado o fio monofilamentar de nylon $\mathrm{n}^{\circ}$ 4-0. A cada 30 minutos foram injetados $5 \mathrm{ml}$ de solução salina $0,9 \%$ na cavidade peritoneal, para hidratação. Terminadas as operações, os animais foram mantidos em gaiolas individuais com água e alimento Labina-PurinaÒ ad libitum durante 5 dias, após os quais os animais foram mortos com superdose de anestésico. Biópsias de ambos os pulmões foram realizadas, sendo as peças fixadas em formol $10 \%$. As lâminas foram processadas por técnica padronizada e coradas com hematoxilina e eosina. As alterações encontradas em cada lâmina dos pulmões examinados foram quantificadas nas intensidades zero (ausente), 1(leve), 2(moderada) e 3(intensa). Foram consi- deradas as alterações: edema, presença de leucócitos nos septos, presença de leucócitos nos alvéolos, hiperemia, atelectasia e pneumonia. A soma das intensidades de zero a 3 de cada uma das alterações representou o escore correspondente a cada animal. Os resultados dos escores foram processados pela análise de variância ANOVA para $\mathrm{K}$ amostras independentes, com $\mathrm{K}$ variáveis numéricas, utilizando-se os testes StudentNeuman-Keuls. Foi considerada significativa a diferença entre os grupos quando $\mathrm{p}<0,05$.

\section{RESULTADOS}

Todos os animais sobreviveram ao experimento e nenhum deles apresentou sinais de infecção da ferida operatória. Comparando-se o exame histopatológico das biópsias pulmonares dos animais jovens com os idosos verificou-se que no grupo $\mathrm{A}$, foram observados escores significativamente menores do que no grupo B. Nos animais jovens não ocorreram alterações pulmonares após as operações que duraram 30 e 60 minutos, surgindo alterações significantes apenas decorrentes de operações que duraram 120 minutos (tabela 1). No que diz respeito ao grupo B, no subgrupo B-1 (tempo operatório de 30 minutos), algumas alterações pulmonares pesquisadas foram observadas, com exceção de leucócitos nos septos alveolares e pneumonia, totalizando o escore 12 (Tabela 2). No subgrupo B-2 (60 minutos de duração) todas as alterações pulmonares procuradas foram verificadas, atingindo o escore 34 . Do mesmo modo, no subgrupo B-3 (operações com 120 minutos de duração) todas as alterações histopatológicas pesquisadas foram observadas, com o escore chegando a 51. No subgrupo de controle apenas 2 animais apresentaram hiperemia alveolar, sendo 1 deles com intensidade 1 e um animal com intensidade 2. Atelectasia de intensidade 1 foi observada em 3 animais. As demais alterações pesquisadas não foram observadas nos pulmões dos controles. Os dados correspondentes a esse subgrupo totalizaram o escore 6 . A análise dos dados mostrou uma diferença significante entre os escores dos subgrupos B-1, B-2 e B-3, quando comparados entre si $(p<0,01)$. Quando foram comparados os subgrupos B-1 e B-2, bem como os grupos B-2 e B3 , as diferenças mostraram-se igualmente significantes $(\mathrm{p}<0,01)$, o que demonstra que quanto mais demorada a operação, maiores as chances de ocorrerem alterações pulmonares em ratos com 18 meses de idade. 
Tabela 1 - Alterações histopatológicas observadas nos subgrupos de animais com três meses de idade. Elas foram expressas em intensidades de 0 a 3 que, somadas, se traduziram em escores.

\begin{tabular}{l|c|c|c|c}
\hline ALTERAÇÕES/Subgrupos & A-1 $(30 \mathrm{~min})$ & A-2 $(60 \mathrm{~min})$ & A-3 $(120 \mathrm{~min})$ & A-4 (controle) \\
\hline Edema & 1 & 3 & 6 & 1 \\
\hline Leucócitos nos septos & 0 & 2 & 7 & 0 \\
\hline Leucócitos nos alvéolos & 2 & 2 & 5 & 0 \\
\hline Hiperemia & 2 & 3 & 3 & 3 \\
\hline Atelectasia & 1 & 1 & 5 & 1 \\
\hline Pneumonia & 0 & 0 & 2 & 0 \\
\hline ESCORES & $6^{*}$ & $\mathbf{1 1 *}$ & $\mathbf{2 8} \mathbf{f}$ & $\mathbf{5}$ \\
\hline
\end{tabular}

* $\mathrm{p}>0,05$ comparado com A-2 e A-4

** $\mathrm{p}<0,01$ comparado com A-3; $>0,05$ comparado com A-4

f $p<0,01$ comparado com A-1, A-2, A-4

Tabela 2 - Achados histopatológicos do grupo B.

\begin{tabular}{l|c|c|c|c}
\hline ALTERAÇÕES/Subgrupos & B-1 $(30 \mathrm{~min})$ & B-2 $(60 \mathrm{~min})$ & B-3 $(120 \mathrm{~min})$ & B-4 (controle) \\
\hline Edema & 3 & 8 & 12 & 0 \\
\hline Leucócitos nos septos & 0 & 7 & 7 & 0 \\
\hline Leucócitos nos alvéolos & 4 & 5 & 10 & 0 \\
\hline Hiperemia & 3 & 7 & 9 & 3 \\
\hline Atelectasia & 2 & 5 & 8 & 3 \\
\hline Pneumonia & 0 & 2 & 5 & 0 \\
\hline ESCORES & $\mathbf{1 2} *$ & $\mathbf{3 4} * *$ & $\mathbf{5 1 ~ f}$ & $\mathbf{6}$ \\
\hline
\end{tabular}

* $\mathrm{p}>0,05$ comparado com B-4; $<<0,01$ comparado com B-2 e B-3

** $\mathrm{p}<0,01$ comparado com B-1, B-3, B-4

f $p<0,01$ comparado com B-1, B-2, B-4

Assim, estabelecendo-se uma comparação entre os escores das duas tabelas, foi observado que entre A-1 e B-1 não houve diferença $(p>0,05)$. Entretanto, entre A-2 e B-2, bem como entre A-3 e B-3 as diferenças foram significantes $(p<0,01)$.

\section{DISCUSSÃO}

A fisiologia pulmonar sofre alterações com o envelhecimento, que podem comprometer a capacidade do pulmão de controlar infecções e outras agressões do meio ambiente. $\mathrm{O}$ tórax do idoso apresenta-se mais rígido em virtude da calcificação das cartilagens costais e da diminuição do espaço entre os discos intervertebrais, o que resulta em menor movimentação do gradil costal. O diâmetro ântero-posterior do tórax está aumentado e a complacência torácica reduzida, já que a própria elasticidade do pulmão também diminui ${ }^{10}$.
Surgem também irregularidades na ventilação alveolar e encarceramento de ar. Há perdas de musculatura bronquiolar nas vias aéreas periféricas, aumento do volume dos ductos alveolares e bronquíolos respiratórios, o que leva à redução do volume e da superfície dos alvéolos ${ }^{11}$. Os idosos são mais vulneráveis à depressão respiratória, em virtude das deficientes respostas à hipóxia e hipercapnia, acompanhadas de menores respostas periféricas e centrais aos quimiorreceptores ${ }^{12}$.

A redução da força e da resistência dos músculos respiratórios, ligada à idade, soma-se às perdas musculares que ocorrem após o trauma cirúrgico, comprometendo as funções respiratórias ${ }^{13}$. A essas alterações somam-se outras, de ordem imunológica, como redução da função ciliar do epitélio respiratório e menor atividade dos linfócitos- $\mathrm{T}^{14}$. Os efeitos clínicos de todas essas alterações são: gradativa redução da PO2 arterial, 
aumento do espaço morto, diminuição do volume expiratório, diminuição da velocidade do fluxo de ar através das vias aéreas, diminuição da função ciliar e diminuição de todos os mecanismos respiratórios de defesa ${ }^{15}$. Essas alterações aumentam muito o risco de atelectasia e de pneumonia no pós-operatório do idoso. No presente estudo em ratos considerados idosos observou-se incidência crescente de alterações histopatológicas nos pulmões, à medida que aumentou o tempo operatório. Optou-se pela utilização de ratos de 18 meses de idade, idosos, baseando-se no fato de que o tempo de vida desses animais dura em média de 16 a 24 meses. Quando as operações duraram 30 minutos, não houve diferença dos escores de ratos jovens e idosos. As diferenças surgiram de modo significativo quando as operações duraram 60 e 120 minutos, levando a crer que o tempo operatório prolongado em idosos pode resultar em prevalência considerável de complicações respiratórias.

Os idosos no pós-operatório têm respiração rápida e superficial, com capacidade vital diminuída por vários dias. A imobilização no leito, os curativos extensos, a dor na ferida operatória e a disfunção da musculatura da respiração, própria do idoso, contribuem para a persistência de baixa capacidade respiratória no pósoperatório. As alterações mais importantes ocorrem após operações torácicas e do abdome superior, quer sejam operações convencionais quer videoendoscópicas $^{4}$. Entretanto, qualquer tipo de operação, quando associada à idade avançada, imobilização, desnutrição e reserva respiratória diminuída, pode criar condições propícias para complicações respiratórias ${ }^{16}$.

Independente da causa específica, a insuficiência respiratória pode ser determinada por colapso alveolar parcial ou completo e edema pulmonar. O colapso alveolar pode ser causado pela respiração superficial do idoso no pós-operatório de cirurgia abdominal ou torácica, pela oclusão parcial ou completa das vias aéreas como broncoespasmo ou na broncoaspiração de suco gástrico. Também ocorre na atelectasia naquelas condições em que ar ou líquido estão ocupando espaço alveolar potencial ${ }^{16}$.

Em se tratando de técnica operatória, o tempo em que uma operação é realizada não deve ser uma procura do cirurgião como se estivesse numa competição de velocidade. O que deve ser procurado, sempre, é a segurança do paciente, a perfeição técnica com manuseio delicado dos tecidos e o trauma mínimo com equipamentos e materiais adequados. Entretanto, no paciente idoso, com todas as características de fragilidade e baixa reserva funcional de todos os órgãos, o tempo operatório tem importância muito grande, apesar de todas as facilidades que os novos equipamentos e drogas anestésicas oferecem. Vários estudos têm mostrado um aumento na incidência de infecção associado com o prolongamento do tempo operatório, mesmo nos pacientes não idosos ${ }^{9}$. O cirurgião, ao indicar uma operação para o paciente acima de 65 anos de idade, além de usar todos os meios disponíveis para prevenir complicações como infecção, tromboflebites, embolia pulmonar, infarto do miocárdio, arritmias, entre outras, deve preocupar-se com o tempo operatório. Em pacientes com idade avançada, operações com duração maior que duas horas acompanham-se de alta incidência de complicações pós-operatórias, especialmente respiratórias, renais e infecção de ferida ${ }^{3}$. Cada hora adicional resulta num acréscimo de $30 \%$ ou mais no índice de complicações pós-operatórias. $\mathrm{O}$ cirurgião que se dispõe a aceitar a responsabilidade de operar um paciente idoso deve ter preparo técnico e emocional para realizar o ato operatório da melhor maneira e no menor tempo possível. A fragilidade do paciente idoso não permite que se perca tempo com indecisões, discussões, descontrole emocional e inseguranças durante o ato operatório. Não restam dúvidas de que se o cirurgião for capaz de realizar, por exemplo, uma gastrectomia tecnicamente bem executada, sem perda de tempo, em duas horas a duas horas e meia, terá um paciente com pós-operatório sem maiores intercorrências. A experiência tem mostrado que pacientes idosos que são submetidos a operações que duram acima de 4 horas e não raro, de 8 a 10 horas, necessitam de tratamento pós-operatório intensivo, freqüentemente de assistência ventilatória mecânica e têm altos índices de morbidez e mortalidade.

\section{CONCLUSÕES}

Os dados disponíveis permitem concluir que o tempo prolongado de cirurgia em ratos com 18 meses de idade contribuiu para o aparecimento e o aumento de alterações histopatológicas nos pulmões. Quanto maior o tempo operatório, maior o comprometimento pulmonar. Nos animais de 3 meses de idade o comprometimento pulmonar somente ocorreu de modo significativo após as operações de 120 minutos de duração.

\section{REFERÊNCIAS}

1. Abyad A. Operative and postoperative evaluation and management in the elderly. Middle East J Anesthesiol 1995; 13:291-306.

2. Behrman SW, Melvin WS, Babb ME. Laparoscopic cholecystectomy in the geriatric population. Am Surg 1996; 62:386-90.

3. Caron JL. Surgery in the elderly: a time for reappraisal. Can J Surg 1996;39:94-5.

4. Cheitlin MD. Coronary bypass surgery in the elderly. Clin Geriatr Med 1996;12: 195-205 
5. Choi JH, Chung HC, Yoo NC. Gastric cancer in young patients who underwent curative ressection. Comparative study with older patients. 1996; 19: 45-8.

6. Edwards AE, Seymour DG, McCarthy JM. A 5-year survival study of general surgical patients aged 65 years and over. Anaesthesia 1996; 51: 3-10.

7. Firilas A, Duke BE, Max MH. Laparoscopic cholecystectomy in the elderly. Surg Endose 1996; 10: 33-5.

8. Koruda MJ, Shelton GF. Surgery in the aged. Adv Surg 1991; 24: 293-331.

9. Cruse PJ, Foord R. The epidemiology of wound infection. A 10 -year prospective study of 62,939 wounds. Surg Cl North Am 1980; 60:27-40.

10. Wahba WM. The influence of aging on lung function. Anesth Analg 1983; 62: 764-76.
11. Levitzky MG. Effects of aging on the respiratory system. Physiologist 1984; 27: 102-21.

12. Buxbaum TG, Schwartz AJ. Considerações perianestésicas no paciente idoso. Clin Cir Am Norte 1994; 74: 45-67.

13. Rizzato G, Marrazini L. Thoracoabdominal mechanics in elderly men. J Appl Physiol 1979; 28: 457-69.

14. Tockman MS. Aging of the respiratory system. In: Katlic MR. Geriatric Surgery. Baltimore, Urban \& Schwarzenberg, 1990, pp 75-84.

15. Sakura S, Saito Y, Kosaka Y. The effects of epidural anesthesia on ventilatory response to hypercapnia and hypoxia in elderly patients. Anesth Analg 1996; 82: 306-11.

16. Seymour DG, Pringle R. Postoperative complications in the elderly surgical patient. Gerontology 1983; 29:262-270.

Medeiros AC, Rocha KBF, Dantas Filho AM, Aires Neto T, Pinto Jr FEL, Medeiros BC. Histopatological consequences of operative time in lungs of old-aged rats. Acta Cir Bras [online] 2003 vol 18 suppl 1. Available in www.scielo.br/acb.

ABSTRACT: The duration of the operations can represent an important factor for a series of postoperative complications, especially in the aged. Objective: study the repercussion in the lungs, of operations of different duration. Methods: Twenty aged rats (18 months old) and 20 young (3 months old) were randomly separated in groups A and B respectively. The groups were divided in A1, A2, A3, A4, B1, B2, B3 and B4, with five rats each. The animals were anesthetized with pentobarbital $(20 \mathrm{mg} / \mathrm{Kg})$ intraperitoneal. In the group $\mathrm{Al}$ and $\mathrm{Bl}$ a 30 minutes operation was done, in the groups A2 and B2 60 minutes, in the A3 and B3 the operation was done in 120 minutes and in the A4 and B4 control groups the animals were not operated. The procedure consisted of laparotomy that was opened and closed so many times as necessary to reach the operative times. After the 5th postoperative day the animals were killed with anesthetic's overdosis and biopsies of both lungs were performed. The biopsies were processed and colored by HE. The histologic findings were transformed in scores for each group. Results: the following results were seen: The groups of the young rats had the scores: $\mathrm{A} 1=$ score $6, \mathrm{~A} 2=11 ; \mathrm{A} 3=28 ; \mathrm{A} 4=5$. Old rats had the following scores: $\mathrm{B} 1=12 ; \mathrm{B} 2=34 ; \mathrm{B} 3=51$ e $\mathrm{B} 4=6$. The statistical analysis revealed significant difference among the scores of the groups A and B. Conclusion: the prolonged time of surgery in rats contributes to significant histopatologic alterations in the lungs. As larger the operative time, larger and more frequent the pulmonary complications.

KEY WORDS: Pulmonary complications. Aged. Operative time. Aged rats.

\section{Correspondência:}

Prof. Dr. Aldo da Cunha Medeiros

Av. Miguel Alcides Araújo, 1889

Natal - RN - 59078-270. 